\title{
Medición de diámetros fustales con relascopio y forcípula finlandesa: efectos en la estimación de volumen
}

\author{
Measurement of upper-stem diameters with Bitterlich mirror relascope and Finnish \\ parabolic caliper: effects in volume estimations
}

\begin{abstract}
CHRISTIAN SALAS E. ${ }^{1}$, MAURICIO REYES Sch., CLAUDIA BASSABER E.
Departamento de Ciencias Forestales, Universidad de La Frontera, Casilla 54-D, Temuco, Chile. Department of Statistics, University of Idaho, PO Box 441104, Moscow, ID 83844-1104, United States.

E-mail: csalas@ufro.cl,csalas@uidaho.edu,mreyes@ufro.cl,cbassab@ufro.cl
\end{abstract}

\begin{abstract}
SUMMARY
The objective of this study was to compare the Bitterlich mirror relascope and the Finnish parabolic caliper in upper-stem diameter measurements, in stem volume estimation and to determine which gives the best results. The study was done in Rucamanque (old growth native forest, IX region, Chile) where thirty-seven trees were randomly chosen. Diameter measurements were taken at 2, 4 and 6 meters above the ground with relascope and Finnish caliper. The "true" or control values were directly taken on the stem with a traditional caliper, using a stairs. The relascope was unbiased for all upper-stem diameters evaluated, however, the Finnish caliper tended to overestimate $(-2,2 \%)$ in the 2 meters height diameter measurements. The stem volume (below 6 meters) estimated with the relascope measurements showed to be unbiased $(-0,5 \%)$. On the other hand, the stem volumes calculated with the Finnish caliper measurements (below 6 meters) were biased $(-2,4 \%)$, although had a higher precision. The efficiency (bias/time of measurement) of the relascope, in order to obtain the necessary variables for stem volume estimations, was significantly higher than the efficiency obtained with the Finnish caliper.
\end{abstract}

Key words: upper-stem diameters, stem volume, relascope, Finnish parabolic caliper.

\section{RESUMEN}

Se comparó la utilización del relascopio de espejos y la forcípula finlandesa en la medición de diámetros fustales y en la estimación del volumen fustal a partir de dichas mediciones, con lo que se determinó cuál instrumento entrega mejores resultados. A treinta y siete árboles de especies nativas del bosque multietáneo del predio Rucamanque (IX Región, Chile), seleccionados en forma aleatoria, se les midieron los diámetros fustales a los 2, 4 y 6 metros de altura con el relascopio y la forcípula finlandesa. Los valores control se obtuvieron mediante medición directa sobre el fuste en pie con forcípula tradicional, usando una escalera de 6 metros. El relascopio entregó estimaciones insesgadas para todos los diámetros evaluados; sin embargo, la forcípula presentó un sesgo significativo $(-2,2 \%)$ en la medición del diámetro a los 2 metros de altura. El volumen fustal hasta los 6 metros, estimado mediante las mediciones efectuadas con el relascopio son insesgadas $(-0,5 \%)$, mientras que con la forcípula finlandesa también se produjo una sobreestimación significativa de $-2,4 \%$; no obstante, con este instrumento se logra una leve mayor precisión. La eficiencia (relación sesgo/tiempo de medición) del relascopio en la obtención de variables necesarias, para estimar el volumen fustal hasta los 6 metros de altura, es un 100\% mayor que con la forcípula finlandesa.

Palabras clave: diámetros fustales, volumen fustal, relascopio, forcípula finlandesa.

\footnotetext{
* Financiado por la Universidad de La Frontera, Proyecto DIUFRO IN-03/98.
} 
BOSQUE 26(2): 81-90, 2005

Medición de diámetros fustales con relascopio y forcípula finlandesa

\section{INTRODUCCION}

Mediante la medición de diámetros fustales de un árbol es posible conocer el ahusamiento, el volumen y la forma de cada individuo que conforman un bosque.

Tradicionalmente, los valores de diámetros a diferentes alturas de los árboles es una tarea compleja de realizar, debido a la dificultad natural de su medición, aun más en especies nativas que presentan formas irregulares. Normalmente a través del volteo de árboles se procede a la medición de diámetros a diferentes alturas. No obstante, existen otras metodologías que han sido utilizadas para cumplir con este objetivo; una de éstas es el uso del relascopio de espejos de Bitterlich, instrumento que permite, además, la medición de altura, pendiente, distancia y área basal del rodal mediante el muestreo puntual horizontal. Desde comienzos de la década de los noventa, están también siendo usados dendrómetros láser, los cuales poseen un elevado costo, hecho que los restringe para su uso masivo en estudios forestales

En este estudio se comparó el uso del relascopio de espejos y la forcípula finlandesa en la medición de diámetros fustales de especies nativas, en condiciones naturales. Así como también su efecto en la estimación de volumen fustal a partir de las mediciones de diámetros realizadas con dichos instrumentos.

\section{MATERIAL Y METODOS}

Area de estudio. El estudio se llevó a cabo en el predio Rucamanque de una superficie de 435,1 ha, situado en los $38^{\circ} 39^{\prime}$ latitud sur y $72^{\circ} 35^{\prime}$ longitud oeste, en la comuna de Temuco, provincia de Cautín, Novena Región de Chile.

El rodal que origina los antecedentes para la presente investigación es un bosque multietáneo, multiespecífico y multiestratificado de Aextoxicon punctatum (Olivillo), Nothofagus obliqua (Roble), Eucryphia cordifolia (Ulmo), Laurelia sempervirens (Laurel), Persea lingue (Lingue). El número de árboles de este rodal es de $586 \mathrm{arb} / \mathrm{ha}, 94 \mathrm{~m}^{2} /$ ha de área basal y $1.178 \mathrm{~m}^{3} / \mathrm{ha}$ de volumen bruto total (1).

Características de los instrumentos empleados. Se empleó el relascopio de espejos de Bitterlich de escala métrica, instrumento bastante versátil y de amplio uso en el ámbito mundial forestal, que permite realizar las siguientes mediciones: área basal del rodal mediante el principio del muestreo puntual horizontal, distancias, alturas, diámetros a diferentes alturas del fuste (diámetros fustales), altura de forma relativa $(f h / d)$, pendiente del terreno y altura del rodal de Hirata (2). En esta investigación, la medición de diámetros fustales es central, ya que representa una clara ventaja para realizar estudios de cubicación de árboles sin necesidad de voltearlos, pues permite la medición simultánea de la altura y el diámetro del tronco a esa altura (3). En Chile, Rivera et al. (4) compararon el uso del relascopio de Bitterlich con otros instrumentos en la medición del diámetro a la altura del pecho en Pinus radiata, pero no en diámetros fustales.

La forcípula finlandesa se ha empleado para medir diámetros fustales hasta los 6 ó 7 metros sobre el suelo en Finlandia, Alemania y Suiza (5). Estos diámetros medidos en altura sobre el fuste son en algunos casos variables independientes en funciones de volumen (6). Este instrumento consiste en un brazo parabólico curvo, el cual es montado sobre una vara telescópica de aluminio. Debido a las características dendrométricas de los árboles que funcionan dentro del bosque adulto multietáneo de Rucamanque, se empleó una forcípula finlandesa de $10 \mathrm{~m}$ de altura, con un brazo parabólico de diámetro máximo de $60 \mathrm{~cm}$, graduada al centímetro. Dicho instrumento fue especialmente diseñado para el presente estudio por la empresa VALDERA S.A.

Para conocer el valor real de los respectivos diámetros fustales a evaluar, se utilizó una escalera de aluminio de 6 metros. Esta escalera puede ser ubicada sobre cada árbol en forma paralela al fuste, gracias a una sujeción mediante cadenas; además, al ser graduada en su longitud y al ubicarse en estas marcas, es posible medir diámetros fustales con la ayuda de una forcípula tradicional o huincha diamétrica.

Selección de árboles muestra. Se seleccionaron árboles en forma aleatoria dentro del rodal, de acuerdo a la participación de las clases diamétricas en el área basal total del rodal. Debido a las características de los instrumentos empleados, los árboles muestra debieron poseer un diámetro con corteza a los $1,3 \mathrm{~m}(d)$ menor a $70 \mathrm{~cm}$, por las 
restricciones de sujeción de la escalera. Además, para evitar problemas de forma se midieron mayoritariamente individuos con buena forma $\mathrm{y}$ sin presencia de lianas de magnitud, por su efecto en la medición con el relascopio.

Medición de los árboles muestra. Para cada árbol muestra se procedió a la medición del $d$ y el diámetro con corteza a los $0,3 \mathrm{~m}$ (dtoc) mediante forcípula tradicional de brazos paralelos.

Primero se midieron con el relascopio los diámetros fustales a los 2,4 y 6 metros $\left(d_{2}, d_{4} y d_{6}\right.$ respectivamente) visando el árbol desde una sola posición a una distancia de 20 metros. No se empleó un trípode para la medición con relascopio, para tratar de imitar las condiciones normales de trabajo en terreno con fines prácticos, así como también, según un estudio realizado por Skovsgaard et al. (7), quienes evaluaron el uso de trípode para dendrómetros láser, el error de medición disminuye sólo en un $2 \%$ al usar un trípode en comparación con el uso de estos dendrómetros en forma manual.

En segundo lugar, se midieron $\operatorname{los} d_{2}, d_{4} y d_{6}$ a través de una forcípula finlandesa de $10 \mathrm{~m}$ de altura y con un brazo parabólico de $60 \mathrm{~cm}$. Para ambos instrumentos se registró el tiempo necesario para realizar dichas mediciones.

Finalmente, se realizó la medición directa de los fustes a través de la escalera, la cual fue posicionada sobre cada árbol en forma paralela al fuste. Esta escalera se graduó con marcas cada $2 \mathrm{~m}$, en donde se efectuó la medición de diámetros con forcípula de brazos paralelos de precisión al centímetro. Se efectuaron dos mediciones de diámetro en forma perpendicular, de las cuales se obtuvo el promedio aritmético. Es importante señalar en este punto que las mediciones efectuadas con relascopio y forcípula finlandesa fueron realizadas por un solo operador, capacitado previamente en la medición con estos instrumentos, y los datos reales (medidos desde la escalera) fueron medidos por otro operador.

Evaluación de los instrumentos. El análisis del comportamiento de cada instrumento se dividió primero en su capacidad de medición de los diferentes diámetros fustales y luego en su capacidad para estimar el volumen bruto fustal del árbol hasta los seis metros de altura. Este volumen fustal hasta los seis metros $\left(v f_{6}\right)$ se obtuvo con las mediciones de diámetro realizadas con el relascopio y forcípula finlandesa. Estos volúmenes estimados se compararon con el volumen real, el cual se obtuvo a través de las mediciones de diámetros fustales realizadas mediante forcípula tradicional desde la escalera. Todos los volúmenes se calcularon utilizando la fórmula analítica de Smalian para cada sección comprendida entre el tocón y los seis metros.

Los datos fueron analizados para comparar el sesgo y precisión. El sesgo, que indica un error sistemático en la predicción, se analizó mediante el sesgo porcentual, el cual fue calculado mediante $(100 *$ [valor real-valor estimado]/valor real). Con el objetivo de evaluar diferencias significativas de los sesgos de ambos instrumentos con respecto al valor ideal cero, se empleó la prueba de t-Student, para un nivel de significancia del 5\%, con contraste bilateral. En la evaluación de la precisión, se modificó lo propuesto por Patterson et al. (8) y Wiant et al. (9) para comparar diferentes métodos de estimación, y se calculó el rango intercuartílico (diferencia entre el cuartil 3 y el cuartil 1) mediante el método de las bisagras de Tukey de los sesgos porcentuales.

La medición de diámetros fustales es una actividad que consume un considerable tiempo y, además, es costosa (10). Por esto es necesario analizar la eficiencia del empleo de los instrumentos como herramientas que ayudan en la estimación del volumen fustal. La eficiencia de éstos no tan sólo debe considerar el sesgo que se obtiene en la estimación de dicha variable, sino que, además, debe contener una idea del costo en que se incurre en la medición de árboles con cada instrumento. En el estudio no se evaluó el costo, pero como una alternativa a esto se empleó el tiempo de medición de un árbol con dichos instrumentos. De esta manera, se calculó el cuociente (sesgo/tiempo) para comparar la eficiencia de ambos instrumentos.

\section{RESULTADOS Y DISCUSION}

Caracterización de la muestra. La estadística descriptiva de la muestra (cuadro 1) indica un amplio rango diamétrico, aunque algo sesgada debido a las características que debían poseer los árboles muestra $(d<70 \mathrm{~cm})$. Esto se debe, en primer término, que a los 2 metros el diámetro 


\section{CUADRO 1}

Estadística descriptiva de los árboles muestra $(\mathrm{n}=37)$.

Descriptive statistic of sampled trees $(n=37)$.

\begin{tabular}{|lcccccc|}
\hline Estadígrafo & $\begin{array}{c}\text { Variable } \\
{[\mathrm{cm}]}\end{array}$ & $\begin{array}{c}h \\
{[\mathrm{~m}]}\end{array}$ & $\begin{array}{c}d_{2} \\
{[\mathrm{~cm}]}\end{array}$ & $\begin{array}{c}d_{4} \\
{[\mathrm{~cm}]}\end{array}$ & $\begin{array}{c}d_{6} \\
{[\mathrm{~cm}]}\end{array}$ & $\begin{array}{c}v f_{6} \\
{\left[\mathrm{~m}^{3} \mathrm{scc}\right]}\end{array}$ \\
\hline Mínimo & 18,5 & 19,0 & 19,0 & 18,5 & 13,0 & 0,158 \\
Máximo & 65,0 & 45,0 & 63,0 & 57,0 & 57,0 & 1,851 \\
Media & 44,8 & 28,4 & 43,0 & 39,6 & 37,7 & 0,915 \\
C.V.(\%) & 29,1 & 17,5 & 28,8 & 28,4 & 30,2 & 52,9 \\
\hline
\end{tabular}

Donde: $h=$ altura total.

del fuste debía ser menor a $60 \mathrm{~cm}$, dado el diámetro máximo de medición de la forcípula finlandesa, y lo segundo hace referencia a las características de sujeción de la escalera. Los valores del coeficiente de variación resultaron bajos aún siendo especies nativas y en bosques altamente irregulares.

A medida que aumenta la altura en el tronco, los diámetros fustales muestran una mayor variabilidad, lo cual sería lógico al analizar que se trata de individuos que han crecido en forma natural y con baja intervención antrópica. Entretanto, el volumen fustal presenta una variación menor a la reportada por Salas (11) para el volumen total individual en Rucamanque, debido a que en el presente estudio sólo se está considerando el volumen de una porción del fuste, con lo cual se disminuye notablemente la variación de esta variable. Además, el volumen fustal es una medida más aproximada a la productividad maderera real de los bosques.

Medición de diámetros fustales. En todos los diámetros fustales evaluados, siempre el relascopio obtuvo un menor sesgo que la forcípula finlandesa (cuadro 2).

A los dos metros, ambos instrumentos sobreestimaron el diámetro; la forcípula posee más del doble de sesgo que el relascopio, en una porción del fuste donde se concentra una parte importante del volumen del árbol. Además, sólo en la medición del diámetro fustal a los dos metros el sesgo porcentual de la forcípula finlandesa presenta diferencias significativas con respecto a cero. Este hecho puede generar diferencias al evaluar las posibilidades de estimar el volumen fustal, mediante las mediciones de diámetros fustales, a partir de los dos instrumentos evaluados.

\section{CUADRO 2}

Sesgo e intervalo de predicción en la medición de diámetros fustales.

Bias and prediction interval for upper-stem diameter measurements.

\begin{tabular}{|c|c|c|c|c|}
\hline \multirow[b]{2}{*}{$\begin{array}{l}\text { Altura de medición del } \\
\text { diámetro fustal }(\mathrm{m})\end{array}$} & \multicolumn{2}{|c|}{ Sesgo (\%) } & \multicolumn{2}{|c|}{ Intervalo de predicción $(\%)^{\mathrm{a}}$} \\
\hline & Relascopio & $\begin{array}{l}\text { Forcípula } \\
\text { finlandesa }\end{array}$ & Relascopio & $\begin{array}{l}\text { Forcípula } \\
\text { finlandesa }\end{array}$ \\
\hline 2 & $-0,6$ & $-2,2 *$ & 6,7 & 10,5 \\
\hline 4 & 1,0 & $-1,1 *$ & 9,9 & 7,6 \\
\hline 6 & 2,4 & $-3,0 *$ & 13,5 & 13,5 \\
\hline
\end{tabular}

* Indica sesgo significativamente diferente de cero $(\mathrm{P}<0,01)$.

a Rango intercuartílico, diferencia entre el cuartil 1 y 3 de los sesgos porcentuales. 
Los sesgos alcanzados por el relascopio son similares a los obtenidos por Kurth y Fischer (12), quienes registraron un sesgo de $1,04 \%$ en la medición del diámetro fustal a los 4 metros de altura. El diámetro fustal medido con relascopio y forcípula presentó una tendencia similar a dicha altura, con un sesgo medio de 1,0 y $-1,1 \%$ respectivamente, aunque la forcípula sobreestima este diámetro.

En cambio, a los seis metros se obtuvo el mayor sesgo para ambos instrumentos con una leve superioridad del relascopio. Esto es lógico, pues a medida que se asciende en la medición del diámetro en el fuste, existe una menor capacidad para visualizar con precisión los bordes de los fustes. Esta tendencia es evidenciada también por Kurth y Fischer (12). No obstante, el relascopio subestima en un $2,4 \%$ y la forcípula finlandesa sobreestima en un $3,0 \%$ a la misma altura, lo cual indica un comportamiento más favorable para el relascopio, por las implicaciones que tienen las mediciones de diámetros en la estimación de volumen.

Siempre las mediciones efectuadas con la forcípula finlandesa tendieron a sobreestimar los diámetros fustales, lo cual se debe al procedimiento empleado para la medición del diámetro con este instrumento, en donde el operador está generalmente obligado en observar hacia arriba para cuantificar el valor de la medición, lo cual normalmente tiende a sobreestimar los valores reales.

Con la forcípula finlandesa, a los 4 metros de altura, se obtuvo un sesgo menor al de los 2 y 6 metros; esto no permitió verificar la clara tendencia evaluada con el relascopio de un aumento del error de medición del diámetro a medida que aumenta la altura fustal.

Para las tres alturas fustales evaluadas, el relascopio posee un sesgo medio de un $0,9 \%$ versus un $-2,1 \%$ de la forcípula finlandesa, vale decir, este último instrumento posee una tendencia clara a la sobreestimación, la cual, de acuerdo a lo evaluado, sería el doble del sesgo del relascopio, el cual, además, subestima.

La dispersión de los sesgos para cada instrumento con respecto a los valores observados (figura 1) muestra un comportamiento libre de algún patrón especial y la mayoría de los sesgos, para todos los diámetros fustales medidos, se concentran entre un rango de $\pm 10 \%$.

Claramente la concentración de los sesgos muestra la tendencia a la sobreestimación en las mediciones efectuadas con la forcípula finlandesa, mientras que el relascopio presenta una dispersión más aleatoria y posee una cierta homocedasticidad. Ahora bien, la dispersión del sesgo aumenta levemente para diámetros fustales de mayores alturas, con la presencia de algunas observaciones que en las mediciones del diámetro fustal a los 6 metros presentan sesgos cercanos al $28 \%$ y $-40 \%$ con relascopio y forcípula finlandesa respectivamente; no obstante estas observaciones, no se ven relacionados con la magnitud del diámetro. En efecto, para verificar la independencia entre los sesgos y los diámetros $d_{2}, d_{4}, d_{6}$ y $d$, se calculó el coeficiente de determinación $\left(r^{2}\right)$, encontrándose valores que oscilaron entre 0,01 a 0,3 , con lo cual se puede inferir que no existe una relación de aumento del sesgo a medida que las dimensiones de los árboles aumentan.

El rango intercuartílico del sesgo (cuadro 2) es el intervalo entre los cuales se concentra el $50 \%$ de los sesgos porcentuales y que, además, están entre los percentiles 25 y 75 . Un rango intercuartílico del sesgo (intervalo de predicción) ideal sería aquel con el menor valor, pues implicaría que los sesgos porcentuales varían levemente alrededor de su media o valor central.

En este contexto, el relascopio en promedio presentó una leve mayor precisión que la forcípula finlandesa. Tal como en el caso del análisis del sesgo, sólo el relascopio presentó una clara tendencia a disminuir su precisión a medida que aumenta la altura de medición del fuste, ya que la forcípula finlandesa presenta una mejor precisión a los 4 metros. Sin embargo, a mayor altura, se iguala la precisión de ambos instrumentos.

De todas maneras, la mayor precisión alcanzada en el diámetro fustal a los 4 metros medido por la forcípula finlandesa puede, en definitiva, causar una mejor precisión en la estimación global del volumen fustal.

Como análisis de las capacidades de predicción de ambos instrumentos, se busca, en primer término, que el sesgo sea lo más cercano a cero, para evitar así errores sistemáticos en las predicciones. Por otro lado, el rango intercuartílico de los sesgos porcentuales indica la precisión de las mediciones. Al considerar ambos aspectos, sesgo y predicción, claramente el relascopio resulta ser un mejor medio para obtener mediciones insesgadas de diámetros fustales y con un buen nivel de precisión. 


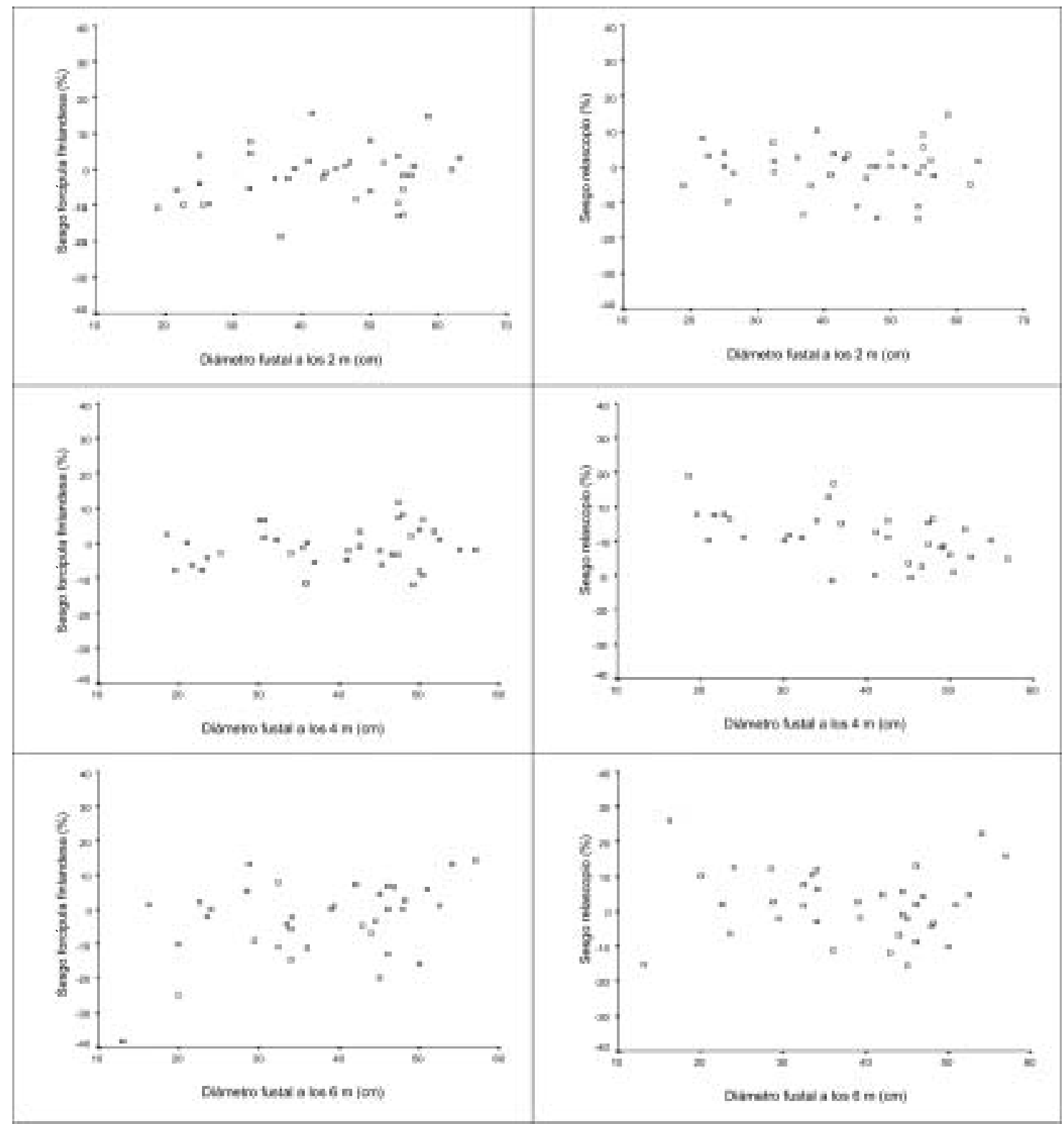

Figura 1. Sesgo en medición de diámetros fustales con forcípula finlandesa (izquierda) y relascopio (derecha). Bias in upper-stem diameters measurements using Finnish parabolic caliper (left) and Bitterlich mirror relascope (right).

Estimación de volumen fustal hasta los seis metros. Tradicionalmente las ecuaciones de volumen son construidas mediante métodos destructivos, los que, conjuntamente con el volteo de los árboles necesarios para la muestra, tienen un alto costo operativo y en el caso de bosques relictos, como es el caso de Rucamanque, presentan también un alto costo ecológico. En este contexto, es importante analizar la viabilidad de emplear las mediciones de diámetros fustales de árboles en pie en la estimación de volumen, para evitar el volteo de árboles. Este aspecto ha sido abordado por diferentes autores, dentro de los cuales es posible destacar los estudios de Kurth y Fisher (12), Lanly (3), Valdés et al. (13), Cailliez (14), Barrena (15) y Salas (11), en donde se han empleado diámetros fustales medidos con relascopio en la estimación de volumen; sin embargo, no se ha analizado la eficiencia que se obtiene al emplear este instrumento para estos fines. 
BOSQUE 26(2): 81-90, 2005

Medición de diámetros fustales con relascopio y forcípula finlandesa

\section{CUADRO 3}

Sesgo e intervalo de predicción en la estimación de volumen fustal (hasta los 6 metros).

Bias and prediction interval in stem volume (below 6 meters) estimations.

\begin{tabular}{|lcc|}
\hline Instrumento & Sesgo (\%) & $\begin{array}{c}\text { Intervalo de } \\
\text { predicción }(\%)^{\mathrm{a}}\end{array}$ \\
\hline Relascopio & $-0,5 *$ & 11,4 \\
Forcípula finlandesa & $-2,4 *$ & 9,8 \\
\hline
\end{tabular}

* Indica sesgo significativamente diferente de cero $(\mathrm{P}<0,01)$

a Rango intercuartílico, diferencia entre el cuartil 1 y 3 de los sesgos porcentuales.

En la estimación de volumen fustal (cuadro 3), realizada a partir de las mediciones con los dos instrumentos, ambos sobreestiman y la forcípula posee un sesgo mayor en más de un $300 \%$ que con el relascopio.

Además, el volumen estimado mediante las mediciones efectuadas con forcípula finlandesa estaría sesgado, difiriendo significativamente de cero $(\mathrm{P}<0,01)$. El sesgo que se obtiene con este instrumento está fuertemente influenciado por el sesgo que se obtuvo en la medición del diámetro fustal a los dos metros, porción del fuste en donde se concentra una importante porción del volumen, sobre todo, en el caso de los árboles estudiados, que poseen grandes dimensiones al com- pararlo con la mayoría de las investigaciones (4, $7,12,13)$. De esta misma manera, a pesar de que con el relascopio sólo se sobreestimaba el diámetro a los dos metros de altura, esta distorsión con el valor real, en definitiva, provoca finalmente que el volumen fustal calculado mediante las mediciones con relascopio también es sobreestimado, debido a la importancia de esta porción del árbol en el volumen fustal.

La precisión de las estimaciones de volumen, que se podrían realizar hasta los seis metros de altura, es mejor con la forcípula finlandesa (el intervalo de predicción es menor), aunque sólo en un pequeño porcentaje. El comportamiento de los errores con respecto a los valores observados (figura 2) muestra una tendencia aleatoria. El $r^{2}$ entre el sesgo del $v f 6$ y el $d$ fue de 0,05 y 0,08 , para la forcípula finlandesa y el relascopio respectivamente, destacando entonces que no existe una relación clara de aumento del sesgo del volumen fustal en árboles de mayores volúmenes.

Para obtener los datos que permitan estimar el volumen fustal de árboles nativos en pie, considerando los resultados aquí expuestos, es recomendable emplear el relascopio, aunque se obtiene una menor precisión en la estimación que con la forcípula finlandesa; dicha estimación se encuentra libre de sesgos.

El relascopio, además, ofrece la posibilidad de emplearlo para la obtención de los diámetros fustales necesarios para cubicar un árbol completo.

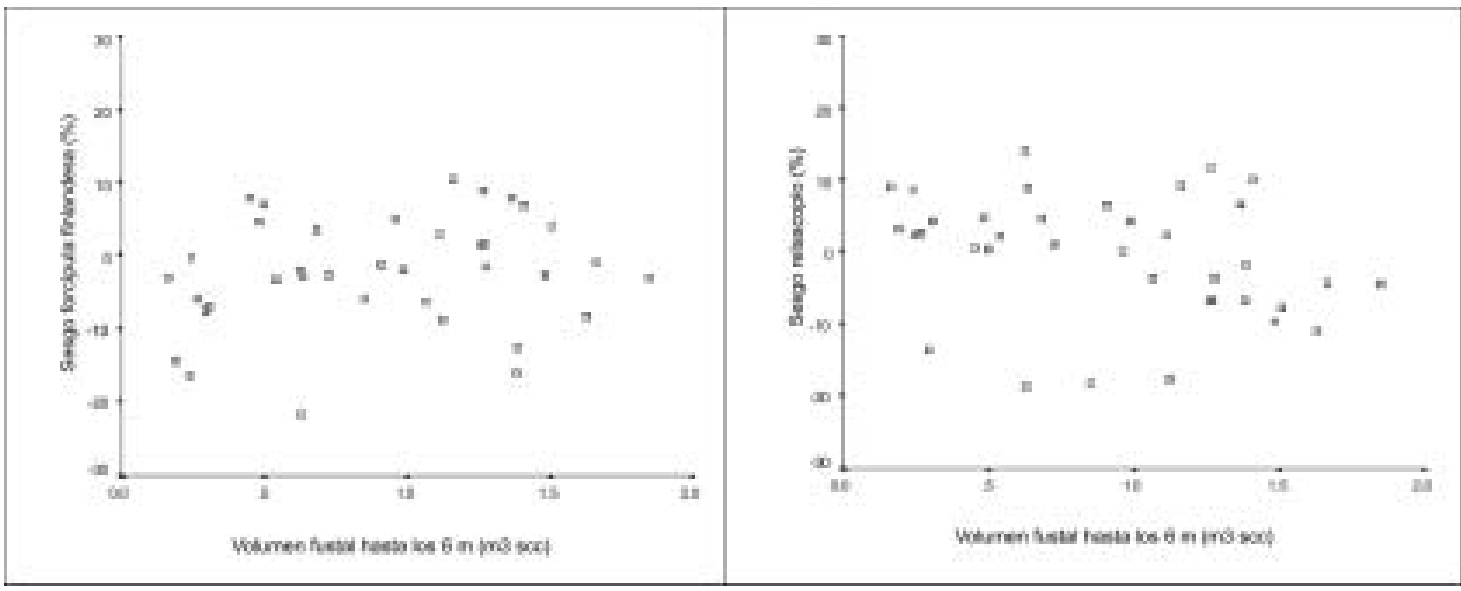

Figura 2. Sesgo en estimación de volumen fustal (hasta los 6 metros) mediante el relascopio y la forcípula finlandesa.

Bias in stem volume (below 6 meters) estimations using Finnish parabolic caliper (left) and Bitterlich mirror relascope (right) 
Por el contrario, la forcípula finlandesa se restringe a ser empleada bajo ciertas condiciones de los árboles y en el caso de la cubicación del árbol completo en pie este instrumento no puede entregar la altura total, la altura comienzo de copa (las cuales se deben medir con otros instrumentos ópticos) y los diámetros a alturas mayores a la cual fue diseñada, lo cual limita su uso, sobre todo en bosques adultos o renovales adultos nativos, en donde árboles de mayores dimensiones son frecuentes.

Análisis de eficiencia de medición de ambos instrumentos. El tiempo necesario para la medición de los tres diámetros fustales $\left(d_{2}, d_{4} y d_{6}\right)$ es un $122 \%$ mayor con la forcípula finlandesa que con el relascopio (cuadro 4), lo cual está influenciado principalmente, debido a que se necesita elevar el brazo parabólico a la altura especificada y posteriormente obtener el valor del diámetro, lo que en definitiva es bastante más trabajo que sólo visualizar mediante el visor del relascopio. Otro aspecto de relevancia es la menor variabilidad del tiempo de medición con el relascopio, lo que demuestra la estabilidad de la estimación en esta variable.

\section{CUADRO 4}

Eficiencia de los instrumentos en la estimación del volumen fustal (hasta los 6 metros).

Efficiency of the instruments in stem volume (below 6 meters) estimations.

\begin{tabular}{|lccc|}
\hline Instrumento & $\begin{array}{c}\text { Tiempo medio } \\
\text { de medición } \\
\text { (minutos) }\end{array}$ & $\begin{array}{c}\text { Sesgo } \\
(\%)^{\text {a }}\end{array}$ & $\begin{array}{c}\text { (Sesgo/ } \\
\text { tiempo) }\end{array}$ \\
\hline Relascopio & $1,8(0,098)$ & 0,5 & 0,3 \\
Forcípula finlandesa & $4,0(0,269)$ & 2,4 & 0,6 \\
\hline
\end{tabular}

Nota: Los valores entre paréntesis ( ) indican el error estándar de la media.

a Expresado en valor absoluto.

Para realizar las mediciones necesarias con las cuales cubicar el fuste de un árbol, el relascopio alcanza una eficiencia un $100 \%$ mayor que con la forcípula finlandesa. Esto significa que mediante el relascopio tan sólo son necesarios 0,3 minutos para lograr un $1 \%$ de sesgo, mientras que con la forcípula son necesarios 0,6 minutos, es decir, para alcanzar el mismo nivel de sesgo en la estimación de volumen se necesita el doble de tiempo con la forcípula finlandesa en comparación al relascopio de espejos, lo que también indica una clara diferencia en los costos de medición.

En este punto es importante señalar que en este estudio no se evaluó el tiempo de desplazamiento que se emplea para trasladarse desde un árbol a otro. De realizarse este análisis y debido a que el desplazamiento con la forcípula finlandesa dentro de los bosques, sobre todo en aquellos que poseen sotobosques densos, es bastante complejo; dicho análisis favorecería aún más el empleo del relascopio.

Este resultado es contrario a lo concluido por Schmid-Haas (1981) (citado por Prodan et al., 16), quien encontró una mayor eficiencia de la forcípula finlandesa frente a otros instrumentos, entre los cuales se encuentra también el relascopio. No obstante, Schmid-Haas evaluó la eficiencia sólo en la medición de un diámetro en altura, el cual pudiera actuar como variable independiente en modelos de volumen, y no considerando varias mediciones fustales que pudieran contribuir directamente a cubicar el fuste de un árbol.

$\mathrm{Al}$ emplear el relascopio como instrumento que entregue todas las variables necesarias para cubicar un árbol en pie, para luego sobre la base de una muestra poder ajustar un modelo de volumen, Valdés et al. (13) reportan que construir una función de volumen local para Pinus radiata mediante el relascopio posee un costo $55 \%$ menor que construirla mediante volteo de árboles y posterior medición con instrumentos convencionales (forcípula de brazos paralelos y huincha de distancia), es decir, en forma tradicional. A juicio de los autores del presente artículo, el costo de ajustar un modelo de volumen de árboles individuales en bosques naturales, a partir de mediciones efectuadas con el relascopio de espejos versus el método tradicional, sería un $48 \%$ menor que con el método tradicional.

Todos los antecedentes expuestos y discutidos, sin lugar a dudas, ratifican al relascopio como un excelente instrumento para poder no tan sólo desarrollar estudios que permitan ajustar modelos de ahusamiento y modelos de volumen con una mayor rapidez y menor costo que con metodologías tradicionales, sino que también poder realizar validaciones de estimaciones efectuadas con 
ecuaciones ya ajustadas en otros sectores, es decir, evaluar la capacidad predictiva de dichas ecuaciones basándose en las mediciones efectuadas con el relascopio (al nivel de diámetro fustal, en el caso de los modelos de ahusamiento, o bien, al nivel de volumen total o fustal, en el caso de modelos de volumen), y si es necesario corregir las estimaciones de los modelos, basándose en las mediciones efectuadas con el relascopio.

Correctas mediciones realizadas a través del relascopio dependen fundamentalmente de una buena capacitación del personal a cargo de éstas, con lo cual también el costo de la captura de datos de terreno podría aumentar, debido a la preparación del personal en el uso de un instrumento bastante más complejo que una forcípula y una huincha de distancia.

No obstante, a pesar de la necesidad de contar con un personal mejor preparado para capturar los datos mediante relascopio, existe un aspecto que es central en la modelación del volumen de árboles individuales, el cual es poder tener una amplia muestra, la que pueda ser segregada en una base para ajuste y otra para el análisis de las capacidades predictivas. Esto se puede lograr gracias al empleo de un instrumento versátil y fácil de transportar en terreno, como el relascopio, que en un mismo periodo de tiempo permite medir una mayor cantidad de árboles, con una leve menor precisión que métodos tradicionales, pero libre de sesgos.

\section{CONCLUSIONES}

El relascopio de espejos entrega estimaciones insesgadas para todos los diámetros fustales; en cambio, la forcípula finlandesa presenta mediciones sesgadas a los dos metros de altura en el fuste. Además, las mediciones efectuadas con forcípula finlandesa presentaron para todos los diámetros evaluados tendencia a la sobreestimación, debido a la forma en que se deben medir los diámetros mediante este instrumento.

Con las mediciones de diámetros fustales obtenidas con el relascopio se verificó la tendencia de un aumento del sesgo, a medida que aumenta la altura fustal, lo cual no fue observado con las mediciones efectuadas con la forcípula finlandesa.

La estimación del volumen fustal hasta los seis metros de altura, obtenida mediante las medicio- nes de diámetros fustales efectuadas con ambos instrumentos, fue insesgada para el relascopio y sesgada para la forcípula.

No existe una correlación significativa entre los sesgos de los instrumentos evaluados y el tamaño de los árboles, lo cual favorece su empleo en bosques multietáneos, en donde normalmente existen individuos de grandes dimensiones.

Se requiere mayor tiempo para obtener las variables, que permiten cubicar el fuste de un árbol en pie, con la forcípula finlandesa que con el relascopio. Entretanto, la eficiencia con la cual se lograría estimar el volumen fustal de un árbol en pie es el doble mediante el relascopio que con la forcípula finlandesa, aunque este último posee una leve menor precisión en las estimaciones.

El relascopio de espejos permite conocer con un buen nivel de precisión el volumen de los árboles en pie, evitando el volteo de árboles, lo cual lo sitúa en una posición ideal para estudiar características dendrométricas en bosques naturales relictos.

\section{BIBLIOGRAFIA}

(1) SALAS, C. Caracterización silvícola de unidades de muestreo permanente de Rucamanque. Informe interno Proyecto DIUFRO N 110201, Departamento de Ciencias Forestales, Universidad de La Frontera. Temuco, Chile. 2002. 25 p

(2) BITTERLICH, W. Das neue Relaskop. Allg. Forstzig. 1958, (Austria), vol. 69, No 23/24, p. 295-299.

(3) LANLY, J. Manual de inventario forestal, con especial referencia a los bosques mixtos tropicales. Organización de las Naciones Unidas para la Agricultura y la Alimentación. Roma, Italia. 1974. 195 p.

(4) RIVERA, J., M. CHEUL, J. LAUSEN, B. BECERRA. Estudio comparativo entre el Spiegel Relaskop y otros instrumentos en la medición de altura y diámetro. Memoria de titulación técnico forestal. Universidad de Concepción. Los Angeles, Chile. 1974. 41 p.

(5) VAN LAAR, A., A. AKÇA. Forest mensuration. Cuvillier Verlag. Göttingen, Alemania. 1997. 418 p.

(6) PARRESOL, B., J. HOTVEDT. Diameter measurement in bald cypress. Forest Ecology Management 1990 (USA), vol. 33/34, p. 509-515.

(7) SKOVSGAARD, J., JOHANNSEN, V., J. VANCLAY. Accuracy and precision of two laser dendrometers. Forestry 1998, (USA), vol. 71, No 2, p. 131-139.

(8) PATTERSON, D., WIANT, H., G. WOOD. Comparison of the centroid method and taper systems for estimating tree volumes. Northern Journal of Applied Forestry 1993, (USA), vol. 10, $\mathrm{N}^{\circ} 1$, p. 8-9.

(9) WiAnt, H., D. PATTERSON, C. HASSleR, G. WOOD, J. RENNIE. Comparison of formulas for estimating volumes of butt logs of Appalachian hardwoods. Northern Journal of Applied Forestry 1996, (USA), vol. $13, \mathrm{~N}^{\circ} 1$, p. $5-7$ 
BOSQUE 26(2): 81-90, 2005

Medición de diámetros fustales con relascopio y forcípula finlandesa

(10) CLUTTER, J., J. FORTSON, L. PIENAAR, G. BRISTER, R. BAILEY. Timber management: a quantitative approach. Krieger Publishing Company. Malabar, USA. 1992. $333 \mathrm{p}$.

(11) SALAS, C. Ajuste y validación de ecuaciones de volumen para un relicto del bosque de Roble-Laurel-Lingue. Bosque 2002, vol. 23, No 2, p. 81-92.

(12) KURTH, H., O. FISCHER. Die Genauigkeit des Spiegel relaskops bei der Ermittlung der Schaftholzformhöhe, der Scheitelhöhe und der Durchmesser in verschiedenen höhen am Schaft. Archiv für Forstwesen 1965, (Alemania), vol. 14, No 11/12, p. 1185-1199.

(13) VALDES, S., VARGAS, V., J. RIVERA. Estudio comparativo entre mediciones convencionales y uso del Relascopio de Espejos en la construcción de una tabla de volumen local. Boletín Técnico $\mathrm{N}^{\circ} 2$, Centro de Cien- cias Forestales, Universidad de Concepción. Chillán, Chile. 1978. 11 p.

(14) CAILLIEZ, F. Estimación de volumen forestal y predicción del rendimiento, con referencia especial a los trópicos. Vol. 1. Estimación de volumen. Organización de las Naciones Unidas para la Agricultura y la Alimentación. Roma, Italia. 1980. 92 p.

(15) BARRENA, V. Estimations de volume de tiges de l'Amazonie péruvienne à partir d'équations de dé filement. Tesis M. Sc. Foresterie et géodésie. Université Laval. Québec, Canadá. 1985. 113 p.

(16) PRODAN, M., R. PETERS, F. COX, P. REAL. Mensura forestal. Serie Investigación y Educación de Desarro1lo Sostenible. Instituto Interamericano de Cooperación para la Agricultura (IICA)/BMZ/GTZ. San José, Costa Rica. 1997. 561 p. 